\title{
Multiple Wavelength Operation of an Erbium-Doped Fiber Laser
}

\author{
Namkyoo Park, Jay W. Dawson, and Kerry J. Vahala
}

\begin{abstract}
Wavelength-locked, six-channel, co-lasing operation using a single gain medium is reported for the first time. The system is an all-fiber, erbium amplifier based design that uses a grating wavelength division multiplexer with a fixed channel spacing of $4.8 \mathrm{~nm}$ for frequency selection. We investigate two possible configurations for the laser cavity.
\end{abstract}

$\mathrm{T}$ HERE is increasing interest in multichannel laser sources at $1.5 \mu \mathrm{m}$ for potential application to optical communication systems using wavelength division multiplexing. Most of the approaches to date use semiconductor lasers, either in arrays of independent gain media [1], [2] or with a frequency control system on a single gain medium [3], [4]. These approaches require precise wavelength controllers as well as independent gain media for each channel, or have difficulties in establishing power balance between channels.

Due to the relatively long fluorescence lifetime of erbiumfiber amplifiers, reduced crosstalk and better stability are expected for simultaneous multichannel operation of an erbium-fiber laser. Here we combine the recently developed erbium fiber laser technology [5], [6] with a fiber-pigtailed, grating wavelength division multiplexer, and demonstrate wavelength-locked, six-channel operation using a single gain medium. To the authors' knowledge, this is the first demonstration of six-channel operation using a single-gain medium, as well as the first demonstration of multichannel operation of a fiber laser.

Fig. 1(a) shows the simplest laser cavity configuration for the multichannel laser. The optical gain was provided by a Corning FiberGain module which has $20 \mathrm{~m}$ of aluminum co-doped erbium-doped fiber pumped by a temperature-controlled $980 \mathrm{~nm}$ diode. The wavelength division multiplexer (WDM) used in the system was a fiber-pigtailed eight-channel grating WDM with an approximate channel spacing of $4.8 \mathrm{~nm}$ and channel bandwidth of $0.8 \mathrm{~nm}$. The insertion loss of the WDM was approximately $6 \mathrm{~dB}$ for each channel.

With variable output couplers made from fiber loop mirrors (FLM) placed as shown in the figure, simultaneous lasing action was possible on all eight channels by careful balancing of the output coupling loss and the gain for each channel. The output powers in this case ranged from 10 to $200 \mu \mathrm{W}$. However, a large power fluctuation was observed (about $3 \mathrm{~dB}$ variation) on a millisecond time scale when each

Manuscript received February 18, 1992; revised March 16, 1992.

The authors are with the Department of Applied Physics, California Institute of Technology, Pasadena, CA 91125.

IEEE Log Number 9200540.

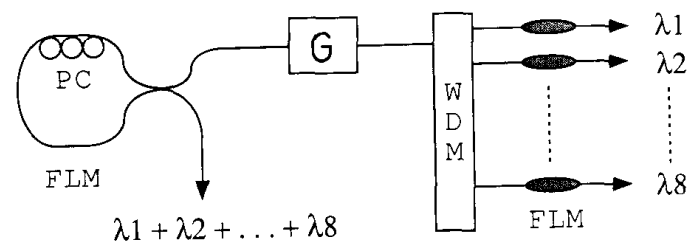

(a)

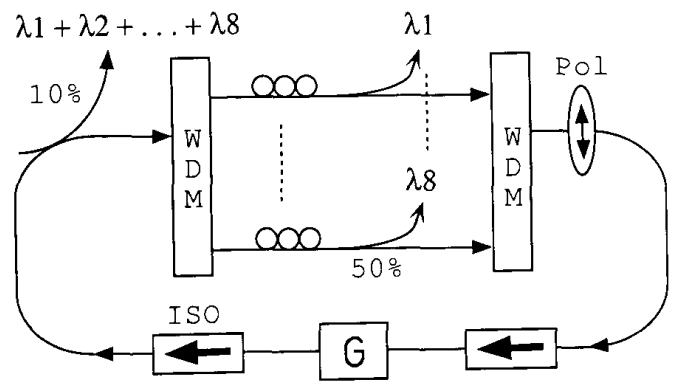

(b)

Fig. 1. (a) Eight-channel laser configuration based on a linear cavity. (FLM : fiber loop mirror, PC : polarization controller, WDM : wavelength division multiplexer, $\mathrm{G}$ : gain module). (b) Eight-channel laser configuration based on a ring cavity (Pol : Polarizer, ISO : optical isolator).

channel output was observed using a photodiode and an oscilloscope. We believe that this fluctuation results from modal instabilities due to spatial hole burning by the intensity standing-wave pattern of longitudinal modes well within the grating passband of each filter.

To test this hypothesis, and to possibly eliminate the problem, we constructed a multichannel, traveling-wave, ring laser by employing a second WDM, wavelength matched to the first (Fig. 1(b)]. In this case, the variable cavity loss was provided by a polarization controller on each channel combined with an in-line polarizer (plasmon wave type, extinction ratio $24 \mathrm{~dB}$ ). Due to a limited number of output couplers, we were able to test only six out of eight channels using this configuration. Simultaneous six-channel operation was possible with the adjustment of intracavity losses of each channel (see Fig. 2). The measured output power was about $50 \sim 150 \mu \mathrm{W}$ for each channel, and the total output power was approximately $1 \mathrm{~mW}$. For this cavity configuration, the power fluctuation was greatly improved. Fluctuations were difficult to measure using the oscilloscope. A full intensity noise characterization measurement is now being conducted. The stability of each channel was relatively insensitive to the total number channels in operation. 


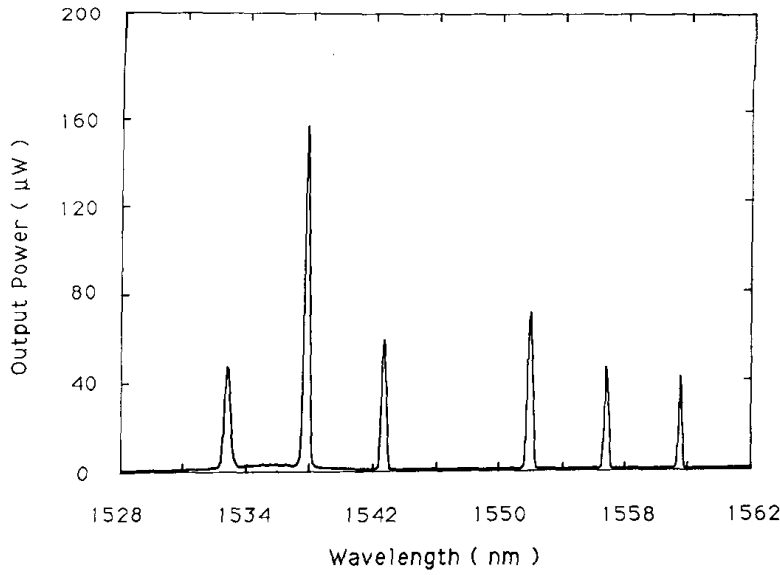

Fig. 2. Spectrum from the multiplexed output port of a six-channel ring laser [Fig. 1(b)]. The output power for the highest peak at $1537.8 \mathrm{~nm}$ was about $150 \mu \mathrm{W}$ at the demultiplexed output port.

The spectrum shown in Fig. 2 was taken from the multiplexed output port of the laser, using a grating monochromator. It clearly shows six-channel operation from 1528 to 1557 $\mathrm{nm}$, with channel separation of $4.8 \mathrm{~nm}$. For this spectrum, the output power for the highest peak $(1537.8 \mathrm{~nm})$ was about $150 \mu \mathrm{W}$ at the demultiplexed output port. It was possible to balance the output powers of all channels at the demultiplexed output ports by adjusting the losses on each channel. Since it was also possible to achieve oscillation from the missing channels in the spectrum $(1542.7 \mathrm{~nm}, 1561.8 \mathrm{~nm})$, it should be straightforward to extend this configuration to eight-channel operation once we have additional components.
To summarize, we have demonstrated stable, wavelengthlocked, six-channel, co-lasing operation in an erbium fiber laser for the first time. Best channel stability was achieved using a traveling-wave laser configuration. Future research will be focused on system improvement by increasing the available number of channels, decreasing channel spacing [7], and further characterization of stability and noise properties.

\section{ACKNOWLEDGMENT}

The authors would like to thank the Northrop Corporation for their support of this work and Dr. W. Masenten for many helpful discussions.

\section{REFERENCES}

[1] I. H. White, "A multichannel grating cavity laser for wavelength division multiplexing applications," J. Lightwave Technol., vol. LT-9, pp. 893-899, 1991.

[2] M. C. Farries, A. C. Carter, G. G. Jones, and I. Bennion, "Tunable multiwavelength semiconductor laser with single fiber output," Electron. Lett., vol. 27, no. 17, pp. 1498-1499, 1991

[3] T. Hidaka and Y. Hatano, "Simultaneous two wavelength oscillation LD using biperiodic binary grating," Electron. Lett., vol. 27, no. 12, pp. 1075-1076, 1991.

[4] G. Couquin, K. Cheung, and M. M. Choy, "Single and multiple wavelength operation of acoustically tuned semiconductor lasers at 1.3 $\mu \mathrm{m}$," IEEE J. Quantum Electron., vol. QE-25, pp. 1575-1579, 1989.

[5] N. K. Park, J. W. Dawson, and K. J. Vahala, "All fiber, low threshold, widely tunable single frequency, erbium doped fiber ring laser with a tandem fiber Fabry-Perot filter," Appl. Phys. Lett., vol. 59, pp. 2369-2371, 1991.

[6] D. A. Smith, M. W. Maeda, J. J. Johnson, J. S. Patel, M. A. Saifi, and A. V. Lehman, "Acoustically tuned erbium-doped fiber ring laser," Opt. Lett., vol. 16, pp. 387-389, 1991.

[7] D. R. Wisely, "32 channel WDM multiplexer with $1 \mathrm{~nm}$ channel spacing and $0.7 \mathrm{~nm}$ bandwidth," Electron Lett., vol. 27, no. 6, pp. $520-521,1991$. 